\title{
Correspondence
}

\section{A systematic review of adults' sitting balance assessments in neurological and neuromuscular conditions}

\section{To the Editor}

We have read with interest the article entitled "A systematic review of adults' sitting balance assessments in neurological and neuromuscular condition" by Alammar \& Alammar published in the Neurosciences journal. ${ }^{1}$ The study discusses new assessment tools to evaluate sitting balance in people with neurological and neuromuscular conditions to be recommended for rehabilitation clinical practice. The review included people with stroke, spinal cord injury (SCI), and multiple sclerosis (MS). As a result, the authors highlighted the clinical importance and the psychometric properties of the Function in Sitting Test (FIST) among people with stroke and MS. However, the authors claimed no access to the article related to the use of the FIST in people with SCI and therefore, did not fully discuss the use and the psychometric properties of the FIST among people with SCI in their review. ${ }^{1}$ We would like to point out that the FIST was used among people with SCI showing appropriate reliability and validity properties.

The paper related to the use of the FIST in people with SCI entitled "Reliability and validity of the function in sitting test among non-ambulatory individuals with spinal cord injury" was published in a peer-review journal "The Journal of Spinal Cord Medicine" and therefore, is accessible for researchers and clinicians. ${ }^{2}$ Through the publisher, the article is available for individual purchase or can be obtained through an institutional membership. A request for access to the article was not received by the corresponding author. It is unclear what barriers the authors faced in an attempt to access the paper. Regardless, the information related to the use of the FIST among people with SCI was not present in this review.

Nonetheless, we would like to highlight that the FIST was satisfactorily used among people with SCI and presented with good psychometric properties and recommended for further exploration in this population. ${ }^{2}$ In brief, the FIST was able to assess all the important components of sitting posture including static, proactive and reactive components with an integration of the sensorial function. ${ }^{2}$ Therefore, the FIST represents an appropriate clinical sitting balance posture assessment among people with SCI. In addition, the FIST presented with an excellent test-retest reliability (ICC $=0.95, p<0.001$ ) and a minimal detectable changeMDC of 4 points. ${ }^{2}$ The FIST also showed a satisfactory internal consistency, Cronbach's coefficient- $\alpha=0.81$ among people with SCI. In addition, the FIST was able to distinguish between high and low injury levels and correlated with the lateral functional reach test $(r=0.64$, $p<0.001)$ showing satisfactory discriminant and convergent validity. ${ }^{2}$ However, due to the heterogeneity of the SCI population according to the injury levels and classification of injury, the authors recommend further exploration of the FIST in people with SCI and suggested modification of the scoring and use of the RASH model to adapt the FIST items among people with SCI. ${ }^{2}$ Recently, further psychometric properties of the FIST were evaluated and the clinical utility of the outcome measure was determined for people with SCI. ${ }^{3}$ The scoring of the FIST among non-ambulatory people with SCI was modified to better reflect the assessment of functional sitting balance in this population. ${ }^{3}$ The authors also reported good validity and reliability properties of the FIST in people with SCI. ${ }^{3}$ The study also determined a cut-off score of 45 or greater to characterize transfer ability in this population with a sensitivity and specificity of $92 \% .^{3}$

In conclusion, the FIST presented with appropriate psychometric properties among people with SCI. The outcome measure can be used to assess sitting posture in clinical settings among people with neurological diseases including stroke, MS and SCI. ${ }^{1,2}$ The FIST might fulfill the need of a simple, easy to administer, quick, and clinically relevant sitting balance assessment among people with neurological diseases including people with SCI.

Libak Abou,

Laura A. Rice,

Department of Kinesiology and Community Health, College of Applied Health Sciences, University of Illinois at Urbana-Champaign, Urbana, Illinois, USA

\section{Reply from the Author}

We received your correspondence to the systematic review title "A systematic review of adults' sitting balance assessments in neurological and neuromuscular conditions". ${ }^{1}$ We are grateful to the input with regards to the FIST uses among individuals with SCI. ${ }^{2}$ We are delighted that you came across the published systematic review. It was highlighted among the limitation of the study the lack of access provided by the Saudi digital library (SDL) and King Abdulaziz City for Science and Technology (KACST). 


\section{Walaa A Alammar, Occupational Therapist, Riyadh, Kingdom of Saudi Arabia}

\section{References}

1. Alammar WA, Alammar HA. A systematic review of adults' sitting balance assessments in neurological and neuromuscular condition. Neurosciences (Riyadh). 2020; 25: 163-168.
2. Abou L, Sung J, Sosnoff JJ, Rice LA. Reliability and validity of the function in sitting test among non-ambulatory individuals with spinal cord injury. J Spinal Cord Med 2019: 1-8.

3. Palermo AE, Cahalin LP, Garcia KL, Nash MS. Psychometric Testing and Clinical Utility of a Modified Version of the Function in Sitting Test for Individuals with Chronic SCI. Arch Phys Med Rehabil 2020; 101: 1961-1972.

\section{Illustrations, Figures, Photographs}

All figures or photographs should be submitted in a high resolution (minimum 300 DPI) electronic version saved in jpeg or tiff format. Original hard copies of all figures may be requested when necessary. Photographs will be accepted at the discretion of the Editorial Board. All lettering, arrows, or other artwork must be done by an artist or draftsman. If arrows are used please ensure they appear in a different color to the background color, preferably black with a white border, or white with a black border. If arrows distinguish different items on the figure then different arrow styles should be used ie. long, short, wide, narrow. Written informed consent for publication must accompany any photograph in which the subject can be identified. Written copyright permission, from the publishers, must accompany any illustration that has been previously published. 